\title{
APPROACHES FOR MAPPING NIGHT-TIME ROAD ENVIRONMENT LIGHTING CONDITIONS
}

\author{
M. T. Vaaja ${ }^{1, *}$, M. Maksimainen ${ }^{1}$, M. Kurkela ${ }^{1}$, J.-P. Virtanen ${ }^{1,2}$, T. Rantanen ${ }^{1}$, H. Hyyppä ${ }^{1,2}$ \\ ${ }^{1}$ Department of Built Environment, Aalto University, Espoo, Finland, (matti.t.vaaja, mikko.maksimainen, matti.kurkela, juho- \\ pekka.virtanen, toni.rantanen, hannu.hyyppa)@aalto.fi \\ ${ }^{2}$ Finnish Geospatial Research Institute, Geodeetinrinne 2, FI-02430 Masala, Finland
}

\section{Commission I, WG VI/6}

\begin{abstract}
KEY WORDS: Imaging Luminance Photometry, Photogrammetry, Road Lighting, Terrestrial Laser Scanning, Mobile Laser
\end{abstract} Scanning, UAV

\begin{abstract}
:
The integration of the 3D measurement techniques with luminance imaging has increased the potential for mapping night-time road lighting conditions. In this study, we present selected static and mobile approaches for the purpose. The measurement methods include conventional 2D imaging luminance photometry and the integration of the luminance imaging with terrestrial and mobile laser scanning. In addition, we present our initial experiences with performing integrated luminance mapping and photogrammetric reconstruction from drone imagery. All of the presented methods require that the camera is calibrated with a reference luminance source. Our results show the results of luminance calibration and feasibility of 3D luminance point clouds for evaluating road surface luminances. In addition, we discuss the other potential applications, limitations and future research.
\end{abstract}

\section{INTRODUCTION}

Road lighting reduces traffic collisions, injuries, and fatalities (Payne et Fenske, 1997; Oya et al., 2002; Plainis et al., 2006; Sullivan et Flannagan, 2007; Wanvik, 2009; Jackett et Frith, 2013; Yannis et al., 2013). However, road lighting must be properly designed, installed, and maintained to be effective. Poorly executed road lighting consumes excessive energy, and does not increase visibility enough to ensure the wanted increase in road safety. Lighting conditions may also change over time due to growth of vegetation and construction in the road environment. Hence, a method is needed to verify the quality of road lighting.

The standardized method for road lighting measurement is imaging luminance photometry. Road lighting measurements are instructed in national and international standards such as ANSI/IES RP-8-14 or CEN/TR 13201:2015 (IES, 2014; CIE, 2015). However, measuring and analysing a three-dimensional (3D) environment with two-dimensional (2D) imaging is rather limited. Furthermore, measuring large road areas easily becomes labor intensive and slow, especially if imaging has to carried out with a tripod.

The night-time conditions set specific requirements for luminance imaging, and planning the camera settings. With static measurements, longer exposure times can be used, which enables the detection of low luminance levels on the road surface. Moreover, static measuring allows high dynamic range (HDR) imaging where images with different exposure times are stacked to capture wide dynamics in one image. With mobile measurements, the exposure times have to remain shorter to allow the movement of the platform. In practice, the exposure time and ISO values need to be adjusted with driving speed in order to maintain a useable signal-to-noise ratio.

The 3D measurement technologies such as terrestrial and mobile laser scanning (TLS, MLS), and unmanned aerial vehicle (UAV) photogrammetry provides effective methods for environmental mapping and monitoring. In particular, TLS has often been used for collecting reference data for other systems, while mobile mapping systems (MMS) have proven their effectiveness for road and other corridor environment mapping (Petrie 2010; Kukko et al., 2012; Yang et al., 2013). In addition, the integration of 3D point cloud with image texture is a common approach in current MMS, which would also allow luminance imaging to be combined with 3D geometry. The use of digital cameras as luminance meters have been introduced by Wüller and Gabele (2007) and Hiscocks and Eng (2013).

This study summarizes possible road surface luminance mapping approaches. The following methods are described: conventional 2D luminance imaging; imaging luminance photometry integrated into TLS; imaging luminance photometry integrated into MLS; and luminance imaging from UAV camera photogrammetry. We present our first results from luminance calibration and the feasibility of these methods for assessing road surface luminances. Furthermore, the future potential of the presented methods is discussed.

\section{METHODS}

\section{1}

\section{Test Sites}

The combination of luminance imaging with TLS was tested in 2013 at Otaranta, Espoo, Finland. The target was a two-lane 100 $\mathrm{m}$ long and $6 \mathrm{~m}$ wide road. The road section was illuminated by five luminaires (high pressure sodium lamp $100 \mathrm{~W}$ ) with a spacing of $32 \mathrm{~m}$ and a height of $10 \mathrm{~m}$. The road was closed during the measurements to avoid traffic.

The combination of luminance imaging with MLS and UAV photogrammetry were tested in 2019 at Munkkiniemenranta, Helsinki, where the work was carried out in a two-lane $6.20 \mathrm{~m}$ wide road section of approximately $800 \mathrm{~m}$ length. As a test area, Munkkiniemenranta was more versatile and larger than Otaranta used in the initial luminance measurements with TLS. Furthermore, this time closing the street from other traffic was 
not a necessity, and the street luminaires at Otaranta were changed after the initial measurements which removed any reason to continue measurements at Otaranta. In Munkkiniemenranta, the luminaires were mounted at an altitude of $8 \mathrm{~m}$ with $33 \mathrm{~m}$ interval between the poles. The MLS, UAV, and the conventional 2D luminance measurement examples were all conducted during the same night. The same area of measurement can be found in each of these three measurements. Hence, they can be compared against each other to a certain extent. Figures 1 and 2 illustrate the measurement locations in Otaranta and in Munkkiniemenranta respectively. In Figure 2, the magenta area represents the area of measurement that can be found in the MLS, the UAV, and the conventional 2D luminance measurement.

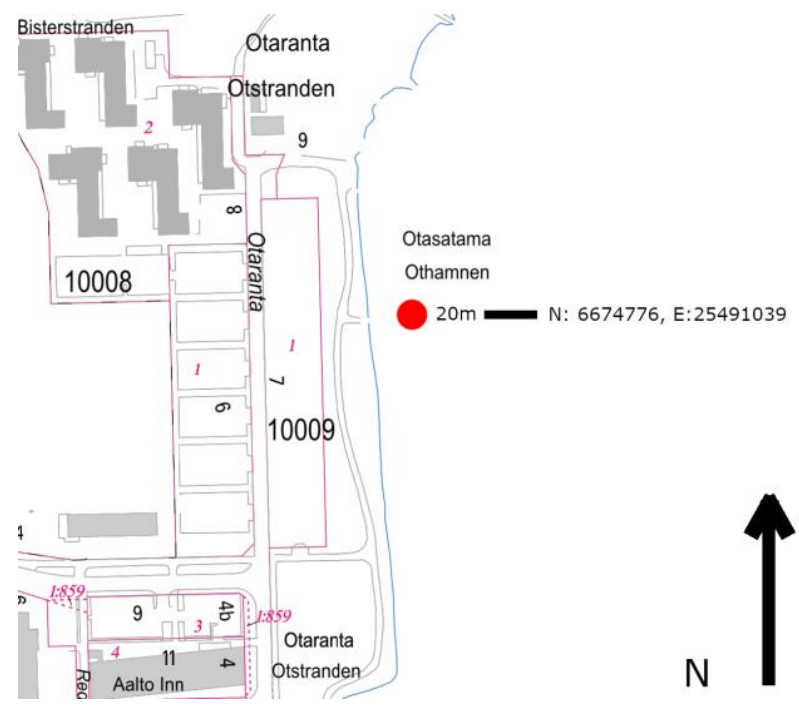

Figure 1. The measurement location in Otaranta.(Espoon karttapalvelu, 2020) (Map set (C) Kaupunkimittausyksikkö, 2020)

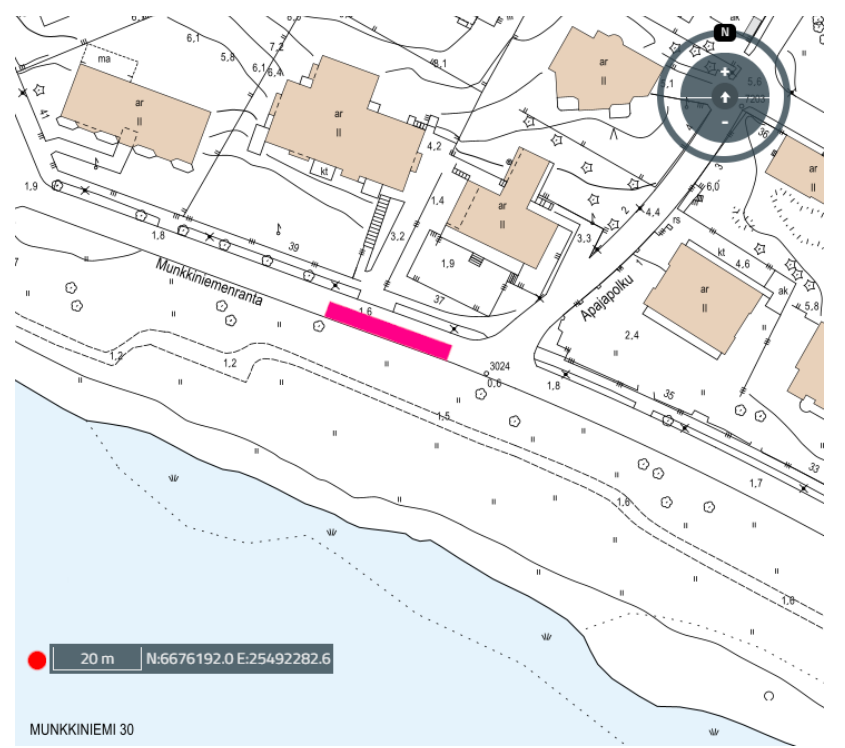

2.2 Photometry

\section{Conventional 2D Imaging Luminance}

2.2.1 Luminance Calculation: The principle behind 2D imaging luminance photometry is the interpretation of digital red, green, and blue (RGB) values as absolute luminance values. In order to do this, a digital camera system needs to be calibrated in terms of sensor sensitivity and lens vignetting. The RGB values can be converted into relative luminance values for example by applying the equation (Equation 1) defined by the International Electrotechnical Commission (IEC, 1999):

$$
L_{r}=0.2126 R+0.7152 G+0.0722 B
$$

where

$$
\begin{aligned}
& L_{r}=\text { relative luminance } \\
& R=\text { red pixel value } \\
& G=\text { green pixel value } \\
& B=\text { blue pixel value }
\end{aligned}
$$

The obtained relative luminance value is then converted into absolute luminance value by applying the camera specific calibration constant and vignetting correction. There are commercially available imaging luminance camera systems such as LMK Mobile Advanced provided by TechnoTeam Bildverarbeitung GmbH or The GL OPTICAM 1.0 luminance measuring camera from Analytik Ltd. A more precise conversion equation would be achieved by characterizing the spectral sensitivity behaviour individually for the used camera system.

2.2.2 Road Surface Luminance Standards: The guidelines for road environment luminance measurement depend on national road lighting design and measurement instructions that are often based on international standard or technical report. In Finland, the national guidelines are based on CEN/TR 13201:2015 technical report (CIE, 2015). The guidelines define three measures for road surface luminance: the average luminance $\bar{L}$, the overall uniformity $U_{O}$, and the longitudinal uniformity $U_{I}$. The area of measurement is defined as the road surface of a single lane between two adjacent luminaires. The measurement is captured at the height of $1.5 \mathrm{~m}$ in the middle of the lane facing the area of measurement in the ongoing direction of the traffic. The distance between the measurement capture point and the first luminaire of the area of measurement is $60 \mathrm{~m}$. The average luminance is the mean luminance value of the area of measurement. Furthermore, the overall uniformity is the ratio between the lowest luminance value within the area of measurement and the average luminance, and the longitudinal uniformity is the ratio between the lowest and the highest measured luminance in the longitudinal center strip of each lane.

\section{3}

Integrating Luminance Measurement into 3D

\section{Measurement}

Figure 3 illustrates the workflow of integrating luminance measurement into 3D measurement. The principle is the same for both TLS, and MLS. The starting point was that all cameras were calibrated with a reference luminance source in laboratory conditions. The workflow also included the calibration of a digital camera for vignetting and geometric distortion (Kurkela et al. 2017).

Figure 2. The measurement location in Munkkiniemenranta.

The magenta area indicates the area of measurement.

(Helsingin karttapalvelu, 2020) (Map set $\odot$ Helsinki City Survey Department, 2020) 


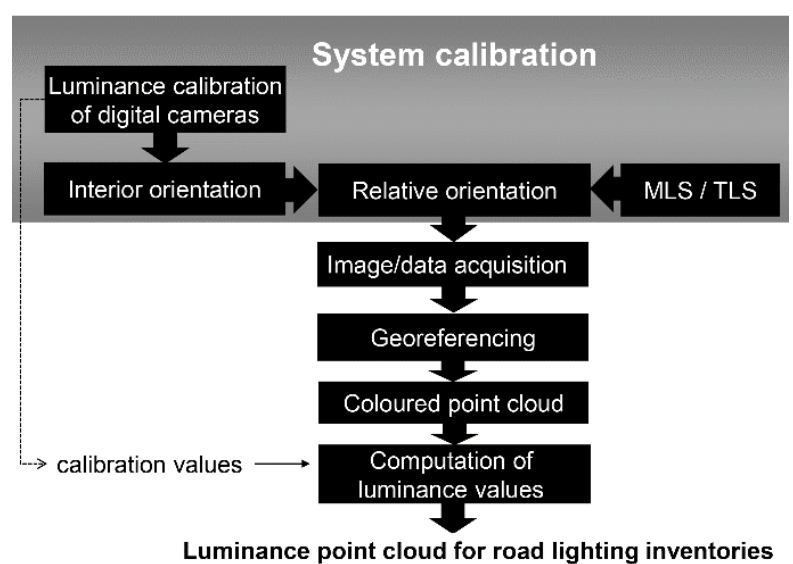

Figure 3. The workflow of combining luminance imaging and laser scanning approaches.

2.3.1 Terrestrial Laser Scanning and Luminance Imaging: For implementing static approach we combined 3D point cloud acquired with a terrestrial laser scanner with luminance imaging. The original study for this approach is presented in Vaaja et al. (2015). A Faro Focus 3D scanner was used to produce the 3D geometry and a Nikon D800E camera for imaging. Alternatively the laser scanner's own camera could be used but we chose an external camera so we could produce better quality for images. The used camera setting for static imaging were aperture of the lens 5.6, exposure time of $8 \mathrm{~s}$ and the ISO value 100 . With these settings, we achieved the measurable luminance range from 0.003 to $2.9 \mathrm{cdm}^{-2}$ (Figure 4).

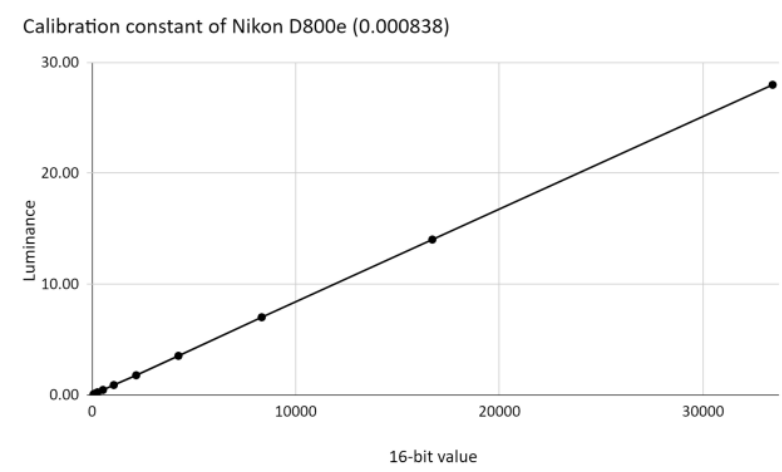

Figure 4. The illustration of Nikon D800e calibration constant. Luminance value in $\mathrm{cdm}^{-2}$.

Data sources were integrated performing the relative orientation between undistorted RGB images and intensity images of terrestrial laser scans (Figure 5). Tie points were detected from every image pair by selecting manually recognizable road objects. On the field, the scanning and image positions were located between the lighting poles which had $30 \mathrm{~m}$ interval. One image was taken for both road directions from one scanning station.
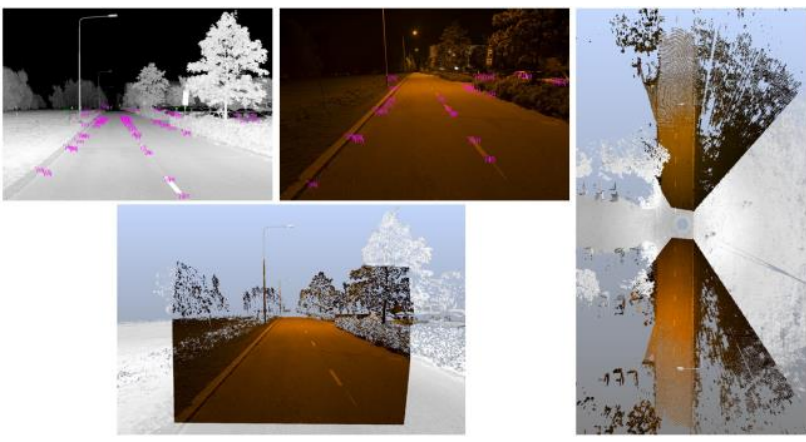

Figure 5. The relative orientation between undistorted RGB images and intensity images of terrestrial laser scans.

2.3.2 Mobile Mapping Systems and Luminance Imaging: We calibrated the LadyBug3 and the LadyBug5 panoramic camera of the Trimble MX2 mobile mapping system in order to map road surface luminances. The original study for this approach is presented in Vaaja et al. (2018). Figures 6 and 7 illustrate the calibration constants of LadyBug3 and LadyBug5 respectively. In this paper, we present results captured using the Trimble MX2 version that had the LadyBug5 panoramic camera in it.

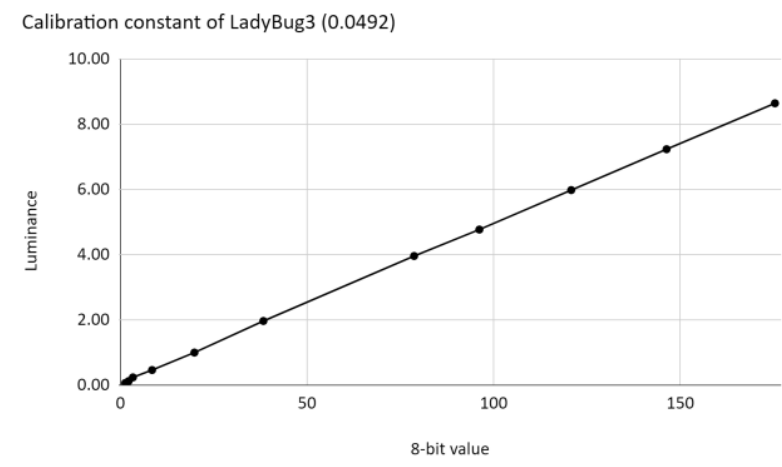

Figure 6. The illustration of LadyBug3 calibration constant. Luminance value in $\mathrm{cdm}^{-2}$.

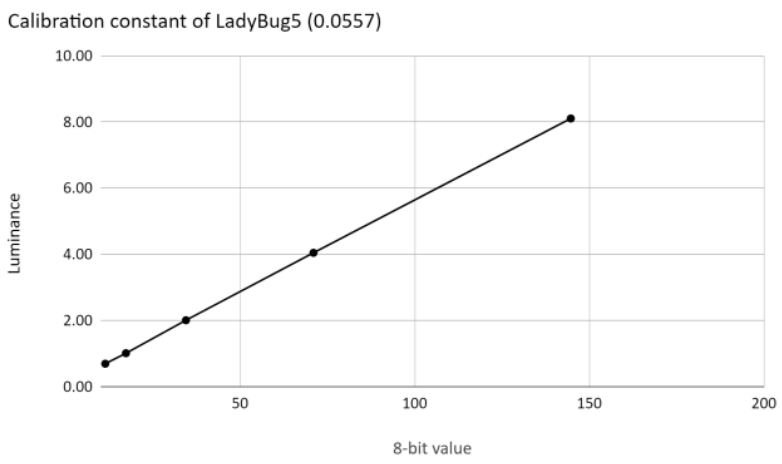

Figure 7. The illustration of LadyBug5 calibration constant. Luminance value in $\mathrm{cdm}^{-2}$.

Trimble Trident software was used for post-processing including the integration of laser scanning point cloud with trajectory data and the interactive orientation for registering the RGB images of Ladybug cameras with the point cloud. 
2.3.3 Unmanned Aerial Vehicle: In this study, we applied DJI Phantom 4 for initial testing of night-time UAV simultaneous photogrammetry and photometry. DJI Phantom 4 is a low-cost consumer level aerial drone. Unlike the cameras used in TLS and MLS luminance imaging, the camera of the UAV had not been calibrated in laboratory conditions. Instead, the calibration constant of the UAV camera was determined by comparing the relative luminance of a detected feature in UAV image to the absolute luminance found in the same feature from MLS measurement. Hence, the integrity and quality of its luminance measurement is not on the same tier as with the TLS and MLS.

\section{RESULTS}

\subsection{Conventional 2D Imaging Luminance Photometry}

We performed conventional luminance measurement to a road section following the measurement guidelines (CIE, 2015). Figure 8 illustrates the pseudo-colored luminance analysis from TechnoTeam LabSoft software. Luminance image was captured using TechnoTeam LMK Mobile Advanced imaging luminance photometer.

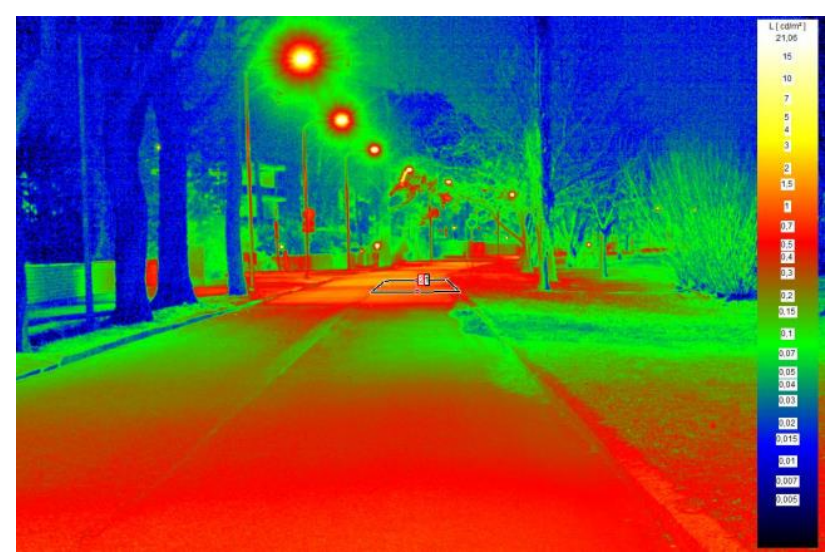

Figure 8. Luminance image in LMK LabSoft with areas of measurement marked at the center.

For this single area of measurement, average luminance, overall uniformity, and longitudinal uniformity were $1.23 \mathrm{cdm}^{-2}, 0.47$, and 0.57 respectively.

\subsection{Luminance Photometry Integrated into Terrestrial Laser Scanning}

Figure 9 illustrates the combination of luminance mapping and TLS. The measurement location (Otaranta) for TLS and luminance imaging combination was different than the location (Munkkiniemenranta) for the MLS, the UAV, and the conventional 2D luminance imaging. Hence, the values measured the TLS are not comparable to the values measured with the other three methods. For a single area of measurement in Otaranta, the average luminance, the overall uniformity, and the longitudinal uniformity were $0.38 \mathrm{cdm}^{-2}, 0.50$, and 0.48 , respectively. The standard deviations varied between 0.029 and $0.036 \mathrm{cdm}^{-2}$ when overlapping luminance values captured from different directions were compared. More detailed statistical analysis of this approach is presented in (Vaaja et al. 2015).

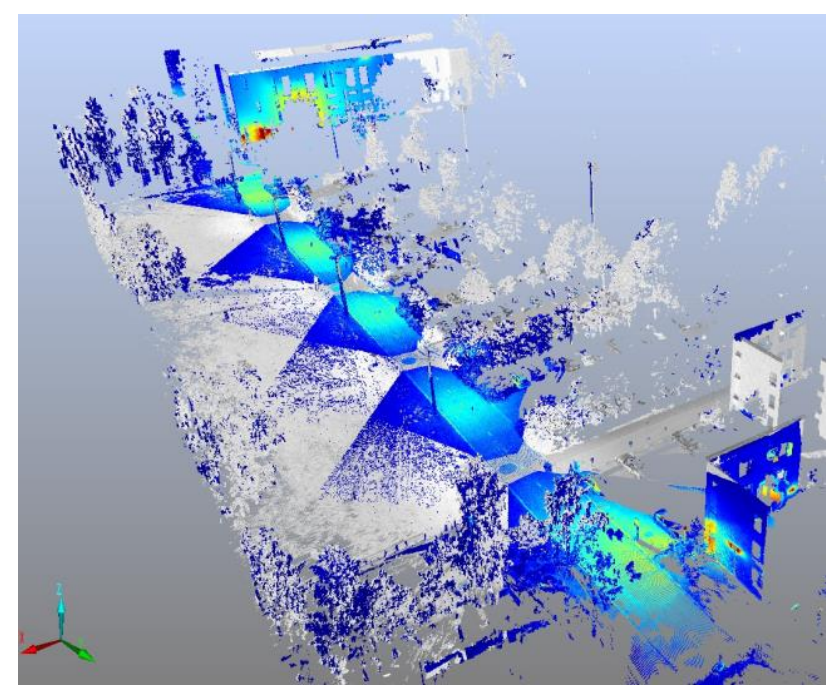

Figure 9. Luminance measurement integrated into TLS.

\subsection{Luminance Photometry Integrated into Mobile Laser Scanning}

Figure 10 illustrates the luminance point cloud captured with MMS. The average luminance at the same area of measurement as LMK was $0.93 \mathrm{cdm}^{-2}$. Furthermore, the overall uniformity and the longitudinal uniformity measured with MMS were 0.69 , and 0.51 respectively.

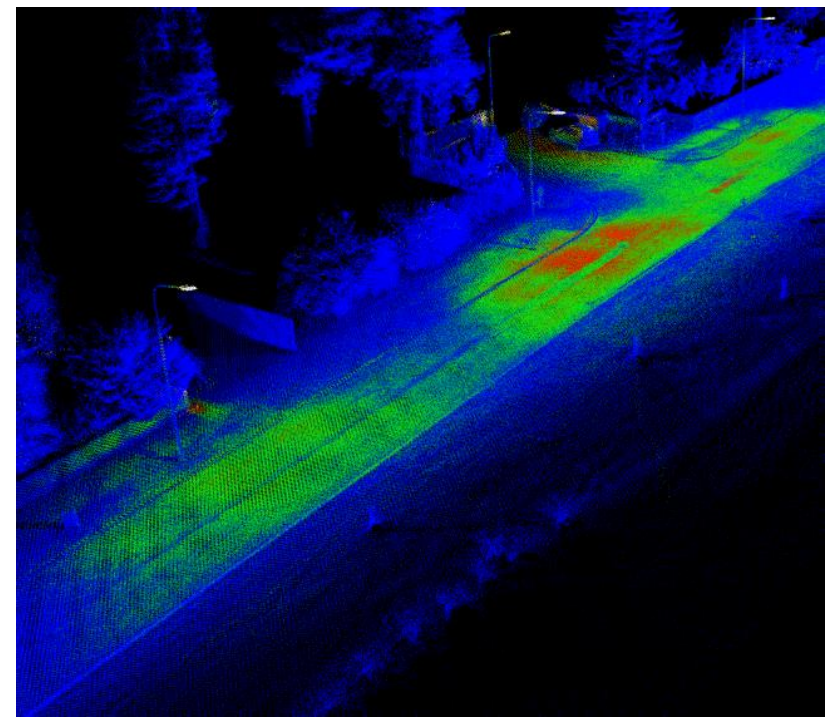

Figure 10. Luminance point cloud from the Trimble MX2 mobile mapping system.

The point cloud luminance data consisted of tens of thousands of points for each area of measurement. Moreover, luminance measurements in low lighting levels are noisy. Hence, an averaging filtering had to be applied to the data in order to obtain utilizable data for average luminance, overall uniformity, and longitudinal uniformity calculation. We applied a median filtering of the luminance values on the XY-plane with the diameter of $1.0 \mathrm{~m}$ around each point in the point cloud. Figure 11 illustrates the effect of median filtering. When overlapping average luminance values measured from different directions and trajectories were compared, the relative difference was at the 10\% level (Vaaja et al. 2018). 


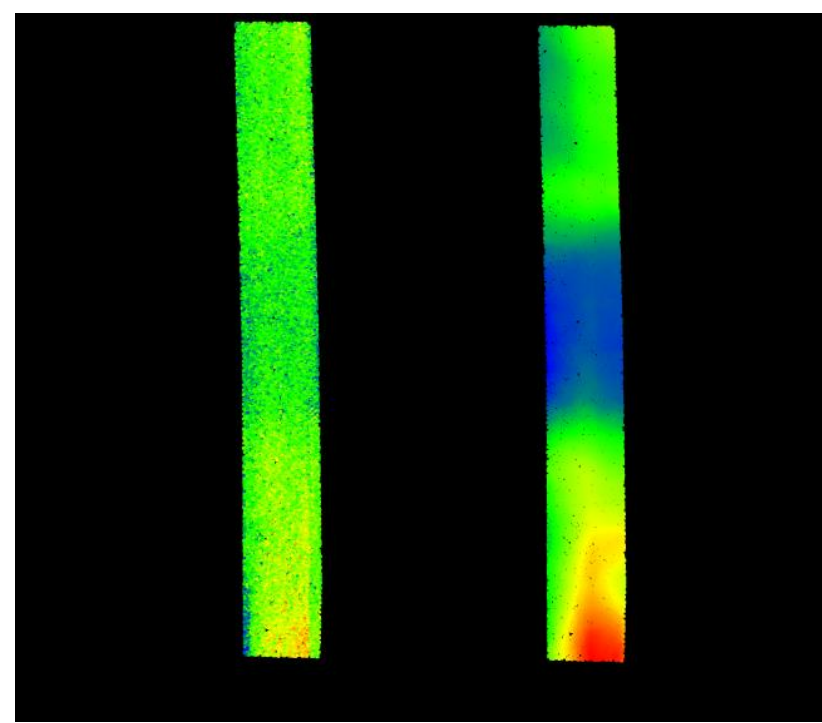

Figure 11. Unfiltered point cloud on the left, and point cloud's luminance values filtered with 1.0 meter radius median filtering on the right.

\subsection{Simultaneous UAV Photogrammetry and Luminance Measurement}

The average luminance at the same area of measurement as with LMK Mobile Advanced was $1.45 \mathrm{cdm}^{-2}$, and the overall and longitudinal uniformities were 0.79 and 0.72 respectively. Figure 12 illustrates the luminance point cloud reconstructed from the UAV imagery.

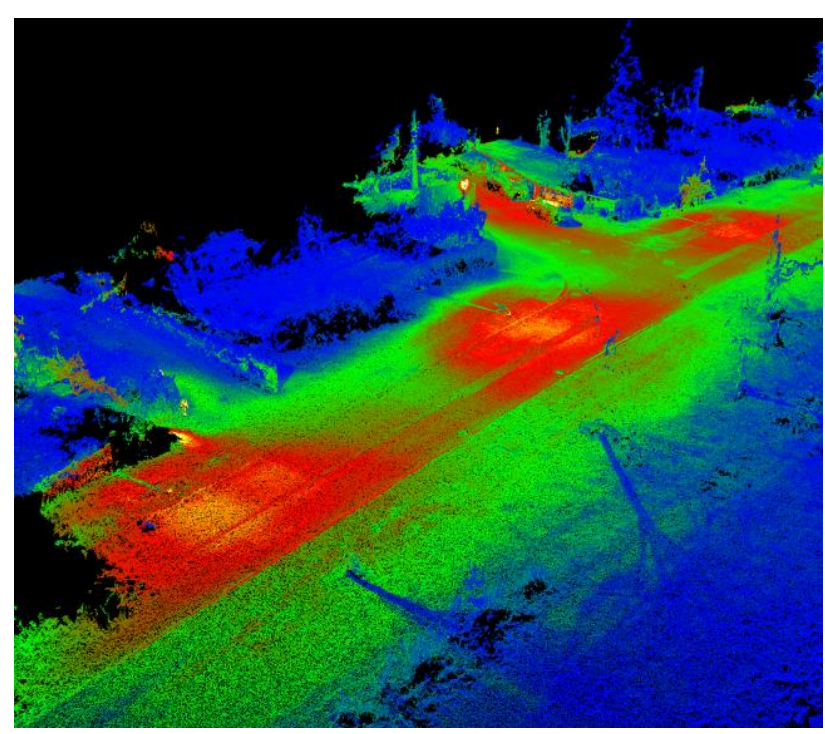

Figure 12. Luminance point cloud from UAV measurement.

Figure 13 illustrates a composition of two nadir images from the photogrammetric block used for the $3 \mathrm{D}$ reconstruction.

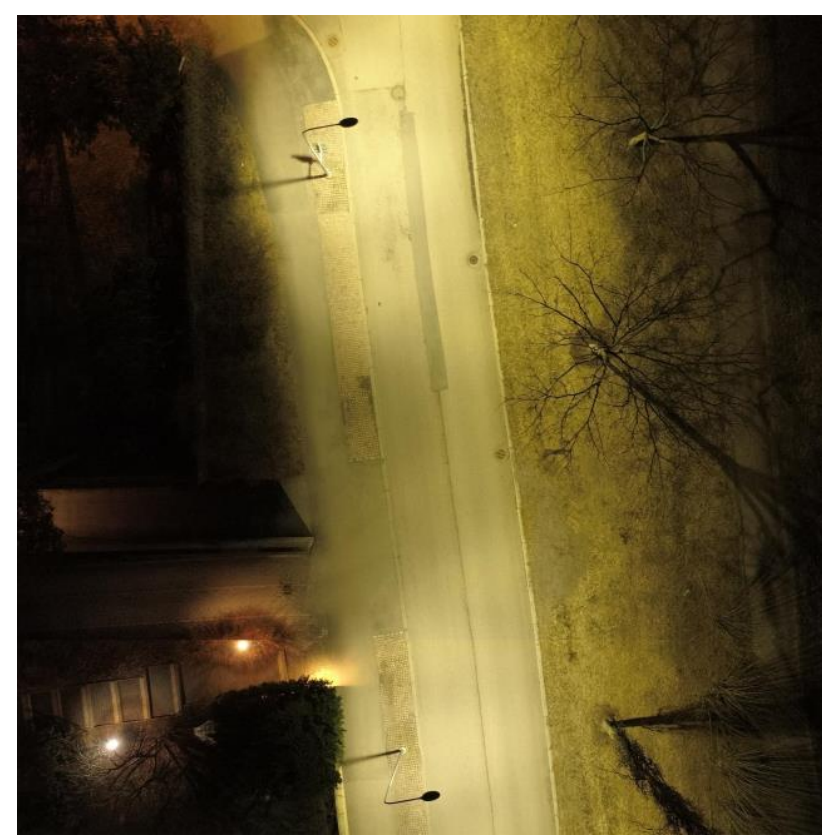

Figure 13. Two combined nadir images captured from UAV at the area of measurement.

\subsection{Comparison of luminance mapping approaches}

As demonstrated by the presented methods, the mapping of a luminance in a night time road environment may be accomplished with several different methods. These approaches produce differing results, both in terms of luminance mapping performance, and other characteristics. The properties of described systems are summarized in Table 1 below. In approached combining LS methods, the geometric accuracy is dependent on their performance.

\begin{tabular}{|lccc|}
\hline System & $\begin{array}{c}\text { Luminance } \\
\text { range }\end{array}$ & $\begin{array}{c}\text { Geometric } \\
\text { precision }\end{array}$ & $\begin{array}{c}\text { Area of } \\
\text { interest }\end{array}$ \\
\hline Static + TLS & not limited & $\begin{array}{c}\text { sub- } \\
\text { centimeters }\end{array}$ & $\begin{array}{c}<500 \\
\text { metres of } \\
\text { road or } \\
\text { street }\end{array}$ \\
MLS & 8 f-stops & centimeters & $\begin{array}{c}\text { Several } \\
\text { kilometres } \\
\text { of road or } \\
\text { street }\end{array}$ \\
$\begin{array}{l}\text { UAV } \\
\text { photogrammetry }\end{array}$ & 8 f-stops & centimeters & $\begin{array}{c}1-2 \\
\text { kilometres } \\
\text { of road per } \\
\text { flight }\end{array}$ \\
\hline
\end{tabular}

Table 1. The properties of selected luminance mapping approaches.

When measuring static luminance measurements, long exposure times and high dynamic range imaging can be used. Hence the luminance range of the luminance measurement is not limited. When measuring luminance from a moving platform, be it a car or a UAV, the luminance range will be limited. When applying the measurement systems presented in this study, the luminance measurement range of a single measurement was $8 \mathrm{f}$-stops or 8 
bits. In absolute luminance values, this was $0.7-8.1 \mathrm{cdm}^{-2}$ for LadyBug5 as an example. This luminance range could be extended if the MMS or the UAV was paired with raw imaging.

Geometric precisions and accuracies of these approaches have been presented in several studies (Kukko et al., 2012; Nex, Remondino, 2014; Schmitz et al., 2019). In Table 1, we present a pragmatic and application-depended approximation for these metrics.

One way to assess the feasibility of a measurement method is by the suitable area of interest of the considered method. For the combination of static luminance measurements and TLS, optimal areas of one measurement session are less than 500 meter long sections of road. TLS and static luminance measurements are slow to conduct, and for safety reasons, it is advisable to close the road section. However, the measurement quality can be the best of these compared methods.

Applying MLS combined with luminance measurement we sacrifice some luminance range and geometric precision, but obtain flexibility and measurement safety. However, the measurement velocities should remain below $25 \mathrm{~km} / \mathrm{h}$, as with higher velocies the motion blurring starts increasingly degenerate the luminance data. Thus, we cannot entirely adjust ourselves to the flow of the traffic. With MMS our area of interest can be several kilometers of road or street within one measurement session.

With UAV, the area of interest can be few kilometers of road with one measurement session. However, UAV is not restricted to follow the road, and thus, can cover outdoor areas and districts. UAV measurements are not occluded by roadside vegetation or infrastructure such as MLS or TLS are. Yet, UAV can be occluded by the canopy when measuring the road surface. Moreover, with UAV it is the most difficult to simulate the position of the road user which is essential in many road lighting measurement applications. Furthermore, depending on the country, the legislation concerning UAVs may cause extra difficulties for their usage.

The optimal method for 3D luminance measurement depends very much on the application. Road areas can be very different. TLS and static luminance measurements are the best for small areas with high priority in terms of safety such as tunnels, junctions, intersections, or bridges. MMSs are the best for the simple road and street areas where efficiency and flexibility are the most appreciated attributes. UAV can be applied for special purposes such as connecting the road lighting measurement to other outdoor lighting measurement, or measuring light pollution.

\section{DISCUSSION}

In this study, we presented a summary of road surface luminance measurement methods.

The results from the currently used 2D imaging luminance photometry method are practically not comparable with the results where luminance values are integrated in point clouds. This is the biggest problem when assessing the novel methods. A benefit of 2D luminance mapping is its consistency when analysing the foveal visual field of the observer in the area of measurement. As stated in the Methods section, in Finland this area of measurement is defined as the single lane road surface between two adjacent road luminaires $60 \mathrm{~m}$ from the observer. Moreover, this can be considered rather limited area of interest, and this constriction may be due to the limitations in the measurement technology. Furthermore, only a small set of picture elements in the luminance image represents a large projection at the road surface.

Luminance photometry can be integrated into TLS to create a luminance point cloud. Furthermore, the luminance measurements can be executed faithfully to the standardized measurement guidelines, and the point cloud can be assembled with sections of road surface points that are registered with the luminance values respectful to the instructed luminance measurements. Following this workflow, the measureable luminance range can be almost arbitrary if high dynamic range (HDR) luminance photometry is applied. However, this process is as slow as the conventional luminance measurement. The improvement gained in this integration is the $3 \mathrm{D}$ visualization and analysis inventory for the luminance values.

When combining luminance photometry with a MMS we lose the integrity with the standardized method, and with current technology, also the measurable luminance range and measurement accuracy decreases. For example, we obtained the measurable luminance range from $\sim 0.2$ to $8.63 \mathrm{cdm}^{-2}$, when we calibrated Ladybug3 panoramic camera for mobile measurements. Furthermore, the MMS luminance measurements are not conducted precisely as instructed in the standard in terms of observer location, and it is not even purposeful to aim at that, as the mobile measurement is a completely different approach. However, with a MMS large road areas can be measured swiftly. Furthermore, camera technology improves continuously, and this narrows the gap in measurement accuracy when compared to static photometry. When using Trimble MX2, the main shortcoming in terms of luminance measurement was the narrow bit depth of Ladybug3 panoramic camera. The 8-bit images support only eight f-stops of dynamic range which limits the luminance measurements. The second downside for Ladybug 3 camera is the sensor sensitivity. Obviously, the camera is mainly intended for daytime use. Therefore, the signal-to-noise ratio hinders the luminance measurement in high gain values of the sensor. Both of these downsides could be overcome by integrating an extra camera into the MMS. A camera that can capture at least $12 \mathrm{f}$ stop dynamic range in a single image, and that has a high signalto-noise ratio. However, in this case the extra camera has to be oriented with the MMS which causes extra labor, and an extra source of uncertainty.

The camera of the UAV used in this study was not calibrated in laboratory conditions. Instead, it was roughly calibrated by comparing the UAV measurement to the MLS measurement. However, this does not mean that the results of UAV measurements would be worthless, as they teach us a lot about the potential of aerial 3D luminance measurements. The simultaneous luminance measurement and photogrammetry executed applying an unmanned aerial system is a very interesting approach for road lighting measurement. As a concept, it is very distant from the conventional luminance measurement as the measurements are captured at locations that are very different compared to the assumed road using observer. For some applications however, UAV luminance measurements might be the best available method. It is also less limited by occlusions than terrestrial methods excluding the occlusion caused by the canopy. Unmanned aerial systems can be automated to quickly measure entireties of built environment. Thus the road lighting measurements can be connected to the outdoor lighting assessment of the road environment. Furthermore, the UAV method could be a remarkable tool for evaluating light pollution, and the correlation between light 
pollution and the canopy of urban vegetation. In comparison to the system presented in this paper, the most obvious improvement to UAV luminance measurement would be integrating a luminance-calibrated professional DSLR camera into the UAV.

Integration of luminance mapping with methods that are capable of acquiring dense 3D point clouds could also support enriching point cloud data sets with further fields of information. The luminance information could also be combined with e.g. visibility analysis, to obtain more holistic measures of visibility conditions of the road environment.

\section{CONCLUSIONS}

During the past two decades, the built environment measurement methods have seen a rapid development. Outdoor lighting is an inseparable part of night-time built environment. Especially in the case of road lighting which has a direct impact to our safety. However, the regulations and standardization concerning the lighting quality measurement have not yet responded to the emerging technologies such as laser scanning, mobile mapping systems, and unmanned aerial systems. We, as the authors of this paper, want to ignite conversations so that the lighting industry would give its response.

\section{ACKNOWLEDGEMENTS}

This research was funded by the Academy of Finland through their grant no 272195 (Center of Excellence in Laser Scanning Research CoE-LaSR), grant no 293389 (project COMBAT) and grant no 323783 (project Quality4Roads). We would like to thank Mitaten $\mathrm{Oy}$ for their cooperation with the luminance measurement equipment.

\section{REFERENCES}

CIE, European Committee for Standardization (CEN). CEN - EN 13201-3, Road lighting - Part 3: Calculation of performance. 2015.

Espoon karttapalvelu (Espoo map service). 2020. Available online: https://kartat.espoo.fi/ (accessed on 29th of January 2020).

IEC, IEC 61966-2-1. Multimedia Systems and Equipment Colour Measurements and Management - Part 2-1: Colour Management - Default RGB Color Space - sRGB, International Electrotechnical Commission: Geneva, Switzerland, 13 pages.

IES, The Illuminating Engineering Society of North America. ANSI/IES RP-8-14, Roadway Lighting. 2014. ISBN \# 978-087995-299-0.

Helsingin karttapalvelu (Helsinki map service). 2020. Available online: http://kartta.hel.fi (accessed on 29th of January 2020).

Hiscocks, P.D., Eng, P., 2013. Measuring luminance with a digital camera: case history.

Jackett, M., Frith, W., 2013. Quantifying the impact of road lighting on road safety-A New Zealand Study. IATSS research, 36(2), pp.139-145.

Kukko A., Kaartinen H., Hyyppä J., Chen Y. 2012. Multiplatform mobile laser scanning: usability and performance. Sensors, 12, 11712-11733.
Kurkela, M., Maksimainen, M., Vaaja, M. T., Virtanen, J. P., Kukko, A., Hyyppä, J., \& Hyyppä, H. (2017). Camera preparation and performance for 3D luminance mapping of road environments. Photogramm. J. Finland, 25(2), 1-23.

Nex, F., Remondino, F., 2014. UAV for 3D mapping applications: a review. Applied geomatics, 6(1), 1-15.

Oya, H., Ando, K., Kanoshima, H., 2002. A research on interrelation between illuminance at intersections and reduction in traffic accidents. Journal of Light \& Visual Environment, 26(1), pp.1_29-1_34.

Payne, D.M., Fenske, J.C., 1997. An analysis of the rates of accidents, injuries and fatalities under different light conditions. Policing: An International Journal of Police Strategies \& Management.

Petrie G. 2010. An introduction to the technology: Mobile mapping systems. GeoInformatics, 13, 32-33, 35-43.

Plainis, S., Murray, I.J., Pallikaris, I.G., 2006. Road traffic casualties: understanding the night-time death toll. Injury Prevention, 12(2), pp.125-138.

Schmitz, B., Holst, C., Medic, T., Lichti, D.D., Kuhlmann, H., 2019. How to Efficiently Determine the Range Precision of 3D Terrestrial Laser Scanners. Sensors, 19(6), 1466.

Sullivan, J.M., Flannagan, M.J., 2007. Determining the potential safety benefit of improved lighting in three pedestrian crash scenarios. Accident Analysis \& Prevention, 39(3), pp.638-647.

Vaaja, M.T., Kurkela, M., Virtanen, J.P., Maksimainen, M., Hyyppä, H., Hyyppä, J. and Tetri, E., 2015. Luminance-corrected 3D point clouds for road and street environments. Remote Sensing, 7(9), pp.11389-11402.

Vaaja, M.T., Kurkela, M., Maksimainen, M., Virtanen, J.P., Kukko, A., Lehtola, V.V., Hyyppä, J. and Hyyppä, H., 2018. Mobile mapping of night-time road environment lighting conditions. Photogrammetric journal of Finland, 26(1), pp.1-17.

Wanvik, P.O., 2009. Effects of road lighting: an analysis based on Dutch accident statistics 1987-2006. Accident Analysis \& Prevention, 41(1), pp.123-128.

Wüller, D., Gabele, H., 2007. The usage of digital cameras as luminance meters. In Digital Photography III (Vol. 6502, p. 65020U). International Society for Optics and Photonics.

Yang, B., Fang, L., Li, J., 2013. Semi-automated extraction and delineation of 3D roads of street scene from mobile laser scanning point clouds. ISPRS Journal of Photogrammetry and Remote Sensing, 79, 80-93.

Yannis, G., Kondyli, A., Mitzalis, N., 2013, October. Effect of lighting on frequency and severity of road accidents. In Proceedings of the Institution of Civil Engineers-Transport (Vol. 166, No. 5, pp. 271-281). Thomas Telford Ltd. 